Cuadernos de Trabajo Social

ISSN-e: 1988-8295

\title{
Introducción a la miscelánea: Presentación del número
}

Teresa García Giráldez

El presente volumen lo integran trece artículos, cuatro proceden de investigaciones con marco teórico y metodológico interpretativo, tres cuantitativo y los restantes son ensayos. En ellos se abordan temas de Trabajo Social, de Servicios Sociales y de Políticas sociales; unos de carácter académico y otros resultado de la sistematización de la práctica profesional. Solo dos artículos contemplan la perspectiva internacional: el autor y el tema que aborda en uno de ellos vincula investigación y profesión en Trabajo Social en el ámbito de la Salud en Brasil; el otro hace referencia a una interpretación crítica de uno de los Pactos de Naciones Unidas en relación con la discapacidad. Los demás artículos se contextualizan en lo nacional, lo regional y lo local español. En relación a la autoría, de los 19 articulistas, 12 son mujeres y 8 varones.

En el primer bloque de artículos se plantea el debate sobre nuevas propuestas de mejora de la calidad de vida, bajo distintas perspectivas: si se trata de un nuevo paradigma o de un enfoque o modelo de intervención alternativo a los residenciales o al que ofrece la familia: se trata de la propuesta del housing first. Los protagonistas del primer ensayo son las personas en situación de pobreza extrema $\mathrm{y}$, del segundo, aquellas que se hallan en situación de dependencia por envejecimiento; de las personas en situación de discapacidad se ocupa el tercero, y se cierra esta reflexión con una nueva mirada crítica a la reglamentación internacional de la discapacidad. También la perspectiva crítica constituye el marco de análisis del primer ensayo citado, siendo en cambio, la racionalidad deductiva el encuadre teorico- metodológico del segundo.

En Las personas sin hogar y la exclusión residencial ¿hacia un cambio de paradigma? Juan Manuel Agulles Martos pone de relieve las oportunidades y los límites de la aplicación de las políticas denominadas Housing First en el contexto actual. Desde una perspectiva crítica plantea este ensayo no solo la dificultad de definir a las personas sin hogar y su representación estereotipada y, lo que es aún más relevante, cómo adentrarse en la pobreza que les embarga. Expone lo difícil que resulta discernir si los modelos que se proponen y las políticas que los inspiran representan un cambio de paradigma en la comprensión de fenómenos, como el del sinhogarismo; o si se trata, más bien, de un modelo alternativo de intervención social. Para avanzar en este discernimiento conviene analizar en qué contexto se proponen, a qué se enfrentan, con qué otros argumentos se justifican y qué disciplinas los arropan. Suponer un cambio de paradigma por la acción social que las políticas de housing first están desarrollando, debería contemplar que se están proponiendo en un momento como el actual y en un contexto sociopolítico determinado, que, al mismo tiempo, está endureciendo las políticas de control del espacio público, dice el autor. Así lo que distingue el cambio de paradigma del enfoque o modelo, como lo analiza Agulles Martos, ha de medirse en términos de ampliación de la adquisición de derechos por parte de las personas transeúntes, como son: el derecho a una vivienda diferente del modelo tradicional de albergue o refugio; el derecho a un techo como mínimo exigible para transeúntes y el derecho a la ciudad. Al autor le resulta difícil considerar que el Housing First permita conquistar este último derecho, que, con los anteriores, justificaría que se considerase un cambio de paradigma. Estos derechos plantean, por lo tanto, nuevos interrogantes sobre la transformación social, necesaria y paradigmática que superen el debate en torno a la mejor forma de gestión de la miseria urbana, y aborden la cuestión del habitar en su sentido más amplio". Apunta el autor que "el derecho a la ciudad, tal y como lo describió Henri Lefebvre", está aún por conquistarse con aquellos modelos. 
Más como modelo alternativo que como cambio de paradigma, y con sus limitaciones, en El bienestar emocional como predictor de calidad de vida en los senior co-housing, Gummá Serra y Castillo Mora señalan la propuesta habitacional co-housing como una oportunidad para las personas mayores, de mejorar su calidad de vida. En esta investigación se ofrece esta alternativa a las tradicionales, residencia o domicilio familiar. Las autoras describen la complejidad y las dimensiones múltiples de lo que entienden por "bienestar emocional" que proporcionaría esta alternativa residencial, al abordar estas variables: emocional, material, personal, físico, jurídico, decisional, relacional e incluyente. Las evalúan con los indicadores que las autoras considerados pertinentes. La comparación entre centros residenciales y la propuesta alternativa cohousing arroja efectos positivos para el bienestar emocional. Resaltan también las limitaciones del acceso a este modelo vivencial, que derivan sobre todo de un elemento no desdeñable: que dependa del status socioeconómico de las personas mayores el acceso a las viviendas colaborativas o co-housing. Ello supondría, como en el caso residencial no público, un condicionante insoslayable para aquellas personas mayores cuyos recursos económicos o socio-culturales son limitados y, por lo tanto, se consideran cercenadas en esta oportunidad. Solo les queda, por lo tanto, esperar hasta obtener una plaza residencial pública o concertada, por no poder acceder a esta propuesta alternativa. El artículo permite contrastar argumentaciones con el ensayo precedente y también con el que viene a continuación.

Desde la perspectiva internacional, Muyor Rodríguez reflexiona, interpreta y analiza, en La (des)institucionaliación en el marco de la Convención Internacional de la ONU sobre los derechos de las personas con discapaci$\mathrm{dad}$, la filosofía y el enfoque de este recurso reglamentario. Con una perspectiva teoricometodológica cualitativa, el análisis crítico del discurso de la Convención y los planteamientos que subyacen en sus generalizaciones sobre la discapacidad, y con un paradigma comprensivo incorpora la mirada de las personas con discapacidad como sujetos activos de derecho. Este paradigma se opone al del modelo de control y enfoque médico-rehabilitador que considera a las personas con discapacidad como "objetos" y la intervención social solo un medio para rehabilitarlas y corregir sus "de- ficiencias". Plantea, como en el caso de Agulles Martos anteriormente analizado, el discurso de derechos que está presente en el documento, lo analiza con otros criterios: la participación social, la visibilidad y la imagen social positiva, y la no discriminación de las personas por su discapacidad. Asimismo interpela a los servicios sociales especializados para que introduzcan estos principios y estas miradas que son, ni más ni menos, los que corresponden al enfoque de derechos humanos de Naciones Unidas, y que han de aplicarlo en su intervención con las personas con discapacidad. Por lo tanto, el autor desmonta las excusas acerca de la institucionalización - "el sujeto dependiente requiere demasiados apoyos, que la familia no puede asumir su rol tradicional y/o que la vivienda no es adecuada"- que encubren un internamiento forzoso y limitador de la autodeterminación de las personas con diversidad funcional. "La autonomía - dice el autor- tiene que ser evaluada en relación con la capacidad individual de tomar las propias decisiones". Esta Convención contempla un modelo social que aleja la mirada institucionalista de la discapacidad-que es consecuencia directa de limitaciones individuales- para centrarla en las limitaciones de la sociedad para asegurar adecuadamente que las necesidades de las personas con discapacidad se tengan en cuenta en las organizaciones sociales.

Un ejemplo del interés por modificar las relaciones interinstitucionales en las organizaciones, lo trata Martínez-López en Nuevas respuestas a la pobreza y exclusión social desde el Trabajo Social: la Mesa ISAE. El objeto de este artículo -escrito también en inglés en este mismo volumen- es analizar una nueva propuesta y una estrategia de trabajo en red a escala local para responder con rapidez a la lentitud de las Administración regional para cubrir las necesidades de la población vulnerable. Desplaza el posible conflicto de la escasez de prestaciones y servicios para atender a las personas en situación de pobreza y exclusión social, hacia medidas acomodaticias de respuesta inmediata. En la iniciativa de una localidad, Elda (Alicante), conocida como la Mesa de Instituciones Sociales en Acción Social de Elda (la Mesa ISAE), contra la pobreza se coordinan, en el periodo analizado de 2010 a 2016, dos niveles de equipos: director y técnico. Se trata de una nueva forma de organización y de trabajo en red entre los tradicionales sistemas organizacionales de los Servicios So- 
ciales de Atención Primaria y al tercer sector (en concreto Cáritas y Cruz Roja). Se trata de dar respuesta de forma coordinada e inmediata a la ciudadanía, una atención urgente, compartiendo la intervención social de casos o la cobertura de necesidades familiares. Se ponen en pie de igualdad las actuaciones de unos y otras, con la finalidad de optimizar sus recursos para atenuar las tendencias de incremento de la pobreza y exclusión social. Se propone como un modelo de atención integral y de desarrollo institucional con "un eje horizontal" para "dar una respuesta integral a las persona". Evoca, a escala micro, el modelo de Fantova Azcoaga, el último artículo de este volumen. El dato de que la atención que presta la Mesa ISAE atienda principalmente a familias biparentales con hijos a cargo y principalmente autóctonas no es baladí.

En este artículo se vislumbra una tendencia presente en la denominada "crisis del estado de bienestar" que argumentaba la necesidad de "desestatalizar" los servicios sociales públicos, en este caso, los Servicios Sociales de Atención Primaria y se naturaliza la derivación de los usuarios en situación de pobreza y exclusión del sector público a las grandes asociaciones no lucrativas, Cáritas o Cruz Roja, para que se gestionen unos recursos y apliquen las intervenciones propuestas por la institución pública. De este modo se va consolidando o se contribuye al desmantelamiento de unos servicios sociales públicos y universales, como es el de Atención Primaria, aunque deficitarios y reformables, hacia un sistema mixto que limita la universalidad.

La reflexión acerca de los principios éticos y la educación en principios, así como su resultado implícito en términos la satisfacción profesional son los temas de los siguientes artículos; ya sea de carácter teórico o aplicado, ya sea en el contexto internacional brasileño o en el nacional español. La educación en derechos humanos y la perspectiva de género, por un lado y la satisfacción laboral de los graduados de esta y otras universidades españolas, por el otro, ocupan los siguientes artículos. El énfasis de dos de ellos se pone en las Universidades, en particular en el de la Universidad Complutense de Madrid y en el profesional de La Rioja.

En el ensayo, El valor de los principios: la ética del Trabajo Social desde la perspectiva del contextualismo pragmatista, Mougán Riveros expone las dificultades de la corriente deontologista y de la utilitarista para resolver los problemas de una ética que cuestiona la racionalidad de los sujetos de la acción social. Analiza las fortalezas de la perspectiva del pragmatismo de Jane Addams y John Dewey, en su intento de articular lo ético con lo político, lo privado con lo público. Ante la necesidad de encuadrar lo social y lo político, la ética contempla dos direcciones distintas: la que se centra en el activismo social (Addams) y la que persigue un proyecto filosófico (Dewey). Ninguna de ellas niega que la perspectiva ética no sea ni social ni democrática; ya que son las sociedades democráticas las que construyen -0 han de hacerlo- las subjetividades acordes con los valores que confieren protagonismo a los ciudadanos, como sujetos activos y responsables de sus acciones. En los actuales Estados sociales de derecho se potencian sobre todo las libertades de los modernos; es decir, no sólo las libertades negativas, sino aquellas que brindan los códigos deontológicos y los principios éticos. Con ellos se potencia el interés y se capacita a los individuos -como actores profesionales o usuarios- para que cooperen entre ellos para buscar soluciones a los problemas sociales, más que para resolver solo los dilemas éticos que se les plantean a los profesionales, como apunta el autor. Otra mirada es la del discurso posmoderno, sin embargo, según la cual los principios y los códigos no es que sean irrelevantes, sino que son corsés que oprimen, conducen a la coerción y anulan la capacidad del sujeto de crear su propia moralidad.

Conviene recordar, que también Mary Richmond, compañera de Addams, buscaba implicar a los usuarios y profesionales del Trabajo Social en la política: "Deberían [los profesionales] también proporcionar argumentos pertinentes que preparan al público para las reformas. En fin, ellas ayudarán más adelante a hacer que las nuevas medidas legislativas sean aplicables en su tarea social" (1962, p.150). Asimismo, la autora vinculaba las reformas con el marco democrático, como única posibilidad de llevarlas a cabo e iba más allá, en relación a la necesidad de interiorizar los principios ético-políticos, añadiendo: "La democracia, sin embargo no es una forma de organización: es un habito cotidiano" (p.166). ${ }^{1}$

Carlos Antonio de Souza Moraes, en La investigación sobre el trabajo profesional del

Richmond, M. (1962). Caso social individual. Buenos Aires: Humanitas. 
asistente social brasileño en el área de la sa$l u d$, avanza resultados de su tesis doctoral. Esta investigación exploratoria cualitativa, desde una perspectiva teórica crítica, analiza la función de la investigación en el quehacer de los trabajadores sociales del área de salud. Constata que en Brasil son escasos los estudios que abordan la relación entre las dimensiones individuales de los trabajadores sociales y los problemas colectivos. Las investigaciones que se han realizado, dice el autor, han contribuido al conocimiento del trabajo profesional; su finalidad "ha servido para mejorar la evaluación, el seguimiento y el mantenimiento del poder institucional"; pero aquellas realizadas, en relación con los usuarios, han contribuido a mejorar el acceso a las políticas, programas y derechos sociales, pero no como un objetivo propio, sino más bien como un objetivo profesional. El motivo de ello es que el ámbito laboral aspira a contar con profesionales operativos, capaces de construir un trato "humanizador" de las relaciones individuales, pero también "responsabilizador" para que los sujetos aborden en primera persona sus necesidades sociales. Las mejoras que proponen las investigaciones en la labor de las trabajadoras sociales, aunque han supuesto un gran esfuerzo de comprensión e investigación acerca de la complejidad de las relaciones sociales, adolecen todavía de bastantes limitaciones. No han sido capaces de generar un cambio social, de crear alianzas y movilizaciones colectivas, dentro y fuera de las instituciones. Dice el autor que, actualmente, en la era de la incertidumbre y la precariedad laboral, sería interesante que los objetivos de las investigaciones se centraran en las dificultades de los trabajadores profesionales para construir vínculos esenciales entre el espacio de trabajo y la realidad colectiva. Hacerlo de este modo implica unas consecuencias políticas para las intervenciones profesionales. De ahí que, por lo general, se limitan al mantenimiento del poder institucional, articulando la responsabilidad del sujeto con el acceso a unas políticas sociales restringidas. Finaliza el autor, diciendo que: "la construcción operativa del hacer (la organización técnica del trabajo), no se ha incorporado de modo prioritario, no se ha integrado, con referentes teorico-críticos comprometidos con propuestas ético-políticas del sujeto profesional".

El artículo, Los efectos de una intervención educativa en materia de derechos humanos con alumnado de Trabajo Social: la valoración de las nociones y el compromiso, forma parte de una investigación doctoral que han realizado Cubillos Vega y Ferrán-Aranaz. Este artículo tiene como finalidad presentar los efectos de un Módulo de Educación en derechos humanos, utilizando como estrategia metodológica un diseño cuasi-experimental, "con grupo control, usando medidas pre y postest, mediante el uso de dos instrumentos validados". Un método cuantitativo que trata de la educación ética y política en derechos sociales, como una pedagogía y una didáctica que acompañarán, a los estudiantes universitarios en sus estudios y a los profesionales en el ejercicio profesional durante su vida. Un estudio longitudinal, cuyos objetivos genéricos "educación sobre, en y para los derechos humanos" - siguen el marco conceptual de la pedagogía crítica y sus propuestas aplicados a distintos niveles: cognoscitivo, procedimental y actitudinal. Se valora la efectividad de esta acción formativa en derechos humanos, al haberlo aplicando a 200 estudiantes de $1^{\circ}$ curso durante el primer semestre del curso. Al comparar los resultados del pre-test en los grupos de intervención y de control con objeto de valorar la evolución, se ha apreciado una situación de partida bastante homogénea, no así en postest, con diferencias más evidentes en términos no solo del conocimiento sino del compromiso, aunque este fuera menos significativo. En la interpretación de los resultados, las autoras afirman que la formación sobre derechos humanos permite aumentar las nociones y el compromiso con ellos; es decir, es un tipo de educación que coadyuva a transmitir conocimiento y a incrementar las actitudes favorables hacia ellos. La aportación de esta experiencia, por lo tanto, promueve su inclusión en los programas de estudio universitarios y las investigaciones de grado y postgrado en diversos contextos, que sistematicen experiencias para ser compartidas y comparadas a nivel global.

También el ámbito universitario madrileño está reflejado en Análisis de género en la educación universitaria en Trabajo Social en España. Díaz Perea y González Esteban reflexionan acerca de la persistencia de la desigualdad de género -de 1985 a 2015-, en el alumnado egresado de las facultades de Trabajo Social españolas y en el personal docente e investigador de la Universidad Complutense. Consideran que las profesiones de cuidado, si bien han 
tenido una función de visibilizar la salida de las mujeres del ámbito reproductivo al productivo, ha favorecido también que accedan a ellas los varones, aunque en proporción más reducida que la de las mujeres. Y ello, a pesar de que la presencia de egresadas y profesionales de Trabajo Social es mayor que la de los varones, habiendo sido el año previo al incremento de tasas académicas (2011-2012) el que mayor número de egresados hubo en las universidades españolas. La brecha numérica entre varones y mujeres que ejercen la profesión en la Universidad Complutense no es tan profunda como la que se experimenta entre el alumnado. Y es que la discriminación que se produce entre el personal docente del sector se refiere en particular a la brecha en relación a los puestos más altos de las jerarquía docente. Y eso es así en la mayor parte de las universidades públicas españolas analizadas en este artículo, por lo que se confirma la permanencia de una particular brecha de género en este ámbito laboral, que es común a otros ámbitos laborales pero difiere de muchos de ellos porque son profesiones naturalizadas como femeninas.

En La inserción laboral y la satisfacción de los egresados del Grado en la Facultad de Trabajo Social de la Universidad Complutense de Madrid, Blanco Carrasco, Sánchez Moreno y Tallón Burgos ofrecen los resultados de un proyecto de innovación docente, realizado entre 2016-2017, sobre los estudios de Trabajo Social en la Universidad Complutense de Madrid y la inserción laboral. Se trata de una investigación metodológicamente cuantitativa cuya finalidad ha sido: por un lado, subsanar una "asignatura pendiente", como es el desconocimiento del destino y la opinión de los egresados de Grado en Trabajo Social sobre la formación recibida y su adecuación a la demanda del mercado laboral y, por otro lado, aportar conocimientos para mejorar la calidad de la titulación. Los participantes son los graduados del curso 2011-2012 hasta el curso 2014-2015, dejando 2 años como el periodo mínimo para encontrar trabajo una vez graduados.

Uno de los resultados más relevantes es el de haber desarrollado una herramienta apropiada para esta titulación. Se trata de un cuestionario que tiene en cuenta las especialidades y las características específicas del mercado laboral de los graduados en Trabajo Social. Las competencias adquiridas, en mayor o me- nor grado e importancia, son apropiadas para desempeñar la labor de intervención social. Entre ellas destacan los autores de la investigación: la resolución de conflictos, la práctica ética, la comunicación eficiente, el aprendizaje activo, las intervenciones reflexivas y los conocimientos actualizados.

El siguiente artículo, La satisfacción laboral de los trabajadores sociales en La Rioja de acuerdo con la teoría bifactorial de Herzberg, de Armas Bravo y Cabezas Medina de los trabajadores sociales con su empleo. Investigan acerca de la perspectiva teórico-metodológica focalizando las competencias adquiridas - cognoscitiva, actitudinales y emocionales- que miden la satisfacción en el trabajo; sus aspectos multidimensionales y multidisciplinares. Definen la satisfacción como capacidad de la persona, sus características personales y psicológicos, para valorar el trabajo que desempeñan los profesionales. Se apoyan en la teoría bifactorial de la satisfacción de Herzberg integrada por: factores intrínsecos y extrínsecos, factores motivadores y organizacionales, que tiene como fundamentos las Escuelas de Desarrollo y de Relaciones Humanas de Mayo. Estos autores consideran que estas dimensiones repercuten en la calidad de las intervenciones $\mathrm{y}$ en los servicios prestados por los diversos agentes sociales. Tenerlo presente es importante para proteger la salud mental de los profesionales. Entre los resultados, los/as trabajadores/as sociales riojanos/as están más motivados/ as por los factores intrínsecos del trabajo que por los extrínsecos. También se mide la satisfacción laboral en el sector público y en el privado, ofreciendo éste además de los elementos desmotivadores, otros que generan mayor satisfacción, como la flexibilidad vinculada con la tarea, la variedad de funciones, mayores responsabilidades y diversificación y ofreciendo un mayor libertad en la toma de decisiones sobre las acciones forma más autónoma.

El tema de la intervención profesional con menores en las situaciones de riesgo y desamparo que dificultan su inclusión social, se abordan en el siguiente bloque de artículos. En el primero se profundiza en el sistema de visitas y en la función de la madre biológica en el proceso de acogimiento familiar; y en el segundo caso, en aquellas violencias que no dejan huellas visibles, perpetradas en el ámbito familiar contra los menores.

La relación familia-menores se aborda en El papel del sistema de visitas en los casos de 
cese de acogimiento familiar: una aproximación desde la perspectiva profesional. Aurrecoetxea Casáus y Pozo Cabanillas exploran las causas por las que se interrumpe improvisamente el proceso de acogimiento. Analizan, a su vez, los elementos que dan estabilidad al acogimiento familiar, a través de la mirada de los profesionales del Servicio de Atención a la Infancia de la Diputación Foral de Bizkaia. La revisión de las investigaciones nacionales e internacionales realizada por las autoras pone el foco en distintos factores: los individuales de los menores, los socio-interactivos filioparentales biológicos y los contextuales con los acogedores; pero no resaltan la importancia de centrar la atención también en los factores relacionados con el proceso del acogida en sí mismo. Las visitas y los contactos de los padres biológicos son, según el parecer de las autoras, aquello que podría conferir una mayor estabilidad al acogimiento familiar. Los principales resultados se agrupan en tres aspectos fundamentales de las visitas: el sistema, la frecuencia y el papel de la madre biológica en las mismas. Lo novedoso de los resultados presentados es la atención especial que se debe prestar al papel de la madre biológica para estabilizar o desestabilizar el proceso de acogida, en particulara atendiendo a los mensajes que emite en las visitas, así como por "su capacidad para construir o recomponer una relación de apego adecuada de cara a una futura reunificación familiar. Resaltan las autoras, por un lado, la necesidad de preparar para el acogimiento a todos los actores implicados. Por el otro, tener presente que las visitas representan una oportunidad para reconstruir los vínculos familiares y para atender a las expectativas, necesidades y los deseos de los adolescentes con las madres biológicas de las que se separan, pero que no desaparecen.

En la investigación cualitativa, de carácter exploratorio, titulada Los menores víctimas de violencia familiar no física: tres visiones complementarias, Sánchez-Morago Hernanz y Becerril Ruiz resaltan las dificultades de profundizar en "una realidad compleja, donde la metodología cualitativa permite conocer unos sentidos y significados que no ofrecen las estadísticas, pero que, a la vez, no tienen la potencia de representación de éstas". Se refieren a la diversidad de riesgos que se ciernen sobre los menores y las dificultades de los profesionales para detectarlos. Se circunscribe a las ciudades de Granada y Valencia. Son riesgos que proce- den de las relaciones violentas con la madre o el padre, violencia entre la pareja o adicciones. También destacan no sólo que la violencia tiene una alta probabilidad de réplica por quienes la padecieron en su infancia; sino que no la valoren como se debe quienes la conocen, la circunscriéndola al ámbito de lo privado; así como la escasa formación en profesionales para su detección. Metodológicamente, han realizado entrevistas a jóvenes-adultos que han sido objeto directo de estas violencias, a un grupo de control y a un tercer grupo de profesionales del ramo, obedeciendo cada una de ellos a objetivos distintos. Sin embargo, a los dos primeros grupos les unen las dificultades para denunciar la violencia, lo que extrema las dificultades de intervención del tercer grupo, el de los profesionales. Estos cuentan con problemas añadidos, como las resistencias de los centros educativos a facilitar información y pruebas de una violencia contra los menores que no deja huellas físicas. Además del sector educativo, también reciben críticas de ineficacia como los Servicios Sociales o el sistema judicial. Los autores proponen trabajar en red con los centros educativos, las familias y los menores, para prevenir estas violencias familiares, realizando "actividades que potencien estilos educativos positivos y doten de información en cuestiones evolutivas que afectan a sus hijos", dicen los autores.

Finaliza la presentación de este volumen con la propuesta que realiza Fantova Azcoaga, en Los agentes en la transformación de los servicios sociales. Con perspectiva sistémica, pormenoriza los elementos, los instrumentos, las políticas y los agentes que han de llevar a la transformación del sistema en el marco de la crisis de los cuidados en la España actual. Para mejorar la organización del sistema de servicios sociales, el autor parte de la equiparación del sistema público al privado, y con ello refuerza el impulso, que está presente en muchas de sus publicaciones, al sistema mixto de bienestar social. Tras señalar los aspectos que desde hace tiempo se vienen señalando para explicar la debilidad del sistema público de bienestar mixto de servicios sociales, explora las tendencias y oportunidades que ofrecería la innovación tecnológica y social a los dos sectores implicados en el quehacer social, si se consigue que funcionen y se articulen los diferentes agentes públicos y privados, en este nuevo contexto del cuidado. Propone lo que denomina la "atención integrada intersectorial" como 
el marco adecuado para unos servicios sociales más acordes con su objeto y con el de otros sistemas (sanitario, laboral, residencial, etc.) en el marco "institucional de gobernanza participativa, multinivel e intersectorial para el bienestar, el desarrollo y la sostenibilidad territoriales y sociales de los servicios de protección social". Ambas innovaciones, la tecnológica y la social, aplicadas al ámbito de las políticas sociales y otras políticas públicas deben caminar juntas, dado que es en los procesos operativos, de gestión y de gobierno, donde muestran su capacidad para articular a los diferentes agentes, como apunta el autor. Esta propuesta supone, por lo tanto, un nuevo reparto de funciones, de interacciones de los agentes: de los políticos, ya sean ideólogos o técnicos, de los académicos y consultores; de las organizaciones profesionales y científicas que los emplean y de la tecnología, como el cuarto agente con gran poder transformador.

Fantova Azcoaga asimila términos y teorías que proceden de los organismos internacionales, y resignifica conceptos con una carga valorativa y unas políticas sociales en sintonía con las propuestas económicas que las orientan. Busca que los asimilen y operativicen el sector público y las organizaciones sociales que cuentan con un gran prestigio en España. Va conformando un estilo de pensamiento, un aparato conceptual que naturaliza un determinado diagnóstico de la situación y un tipo de alternativa. Se trata de una visión de la realidad que actúa como verdadero tapón para la elaboración de propuestas contrahegemónicas. Evita, por un lado, la confrontación política, adoptando un debate que se sitúa en cuestiones técnicas y metodológicas, eludiendo de manera cuidadosa su identificación con encuadres ideológicos y políticos. Esta propuesta se va instalando de manera sutil en los servicios sociales, no se perfila como parte de unas políticas sociales en pugna, sino que se va naturalizando la adopción de medidas sin que vayan destinadas a la reducción de las desigualdades y la precariedad que genera el sistema. Se centra en la acción de cuidar, pero se ha de suponer a quién, para qué y para quién ya que no hay en su artículo ninguna referencia a las personas, a los conflictos, derivados en gran medida de las desigualdades estructurales, no exclusivamente personales, y que en su día se contemplaron sectorial y colectivamente. Las respuestas públicas, como derechos sociales más o menos condicionados, de universalidad limitada, supusieron un alivio asistencial, sí, pero un alivio para los usuarios. En su lugar, el autor abunda en los que proporcionarán - ¿a quién?- las tecnologías e innovaciones de unos "servicios sociales personalizados, preventivos, comunitarios, integrados, ecológicos, interdisciplinares". ¿Contribuirán a hacer invisibles las causas estructurales que no se afrontaron en su día, ni parecen interesar ahora, salvo por algunas de sus consecuencias, en términos de racionalidad, eficacia y eficiencia para abordar la crisis de los cuidados? 
